\title{
Fluid Motion of the Outer Core in Response to a Temperature Heterogeneity at the Core-Mantle Boundary and Its Dynamo Action
}

\author{
Shigeo Yoshida* and Yozo Hamano \\ Department of Earth and Planetary Physics, Faculty of Science, \\ University of Tokyo, Yayoi, Bunkyo-ku, Tokyo 113, Japan
}

(Received December 1, 1992; Revised July 28, 1993; Accepted August 5, 1993)

\begin{abstract}
We perform a simple linear analysis of the response of the outer core fluid to a sectorial temperature heterogeneity at the core-mantle boundary (CMB). The locations of upwellings and downwellings are shown to be controlled by the Elsasser number. When the Elsasser number is less than the order of unity, upwellings occur to the east of hot regions, and downwellings occur to the west of hot regions. When the Elsasser number is more than the order of unity, upwellings occur beneath hot regions, and downwellings occur beneath cold regions. Our theory provides an explanation for the correlation between the observed magnetic feature and the lateral heterogeneity of the seismic velocity in the lower mantle, which would correspond to the temperature heterogeneity at the CMB. From the correlation, we infer that the Elsasser number is a little less than unity in the Earth's outer core. Moreover, we investigate whether the resulting flow can sustain dynamo action or not. A temperature heterogeneity of the order of $10^{-6} \mathrm{~K}$ over a length scale of $10^{3} \mathrm{~km}$ would be sufficient for the generation of dynamo action.
\end{abstract}

\section{Introduction}

Thermal interaction between the mantle and the core has attracted much attention since BloxhAM and GubBins (1987) stressed its importance. They found a correspondence between the pattern of the magnetic field and that of the heterogeneity of the lower mantle, and attributed it to thermal coupling. They proposed a simple relation that flow goes upward beneath hot regions of the lower mantle and sinks beneath cold regions. In rotating systems, however, thermal response is not so simple; for example, ZHANG and GUBBINS (1992) found that upwellings and downwellings occur between hot and cold regions in a non-magnetic system.

Here we consider the thermal response of the outer core with the effects of both rotation and the magnetic field taken into account. Both of these effects are essential for considering the dynamical behavior of the fluid outer core, and cannot be separately investigated. We studied the linear response of the outer core fluid to a lateral temperature heterogeneity on the CMB. Since our main purpose is to clarify the physical mechanism of the thermal response under the influence of rotation and the magnetic field, we simplify the system, only the two effects being taken into account.

The linear analysis is performed in a simple system similar to that used by Busse $(1970,1975$, 1976) for the problem of thermal convection. The outer core is approximated by an annulus with inclined top and bottom boundaries. The simplicity of the system enables us to obtain analytical solutions and clear physical perspective. The mathematical procedure we used is essentially the same as Busse's, but the situation is different; we consider the convection driven by horizontal temperature difference, while Busse treated that driven by unstable vertical temperature gradient.

*Present affiliation: Earthquake Research Institute, University of Tokyo, Yayoi, Bunkyo-ku, Tokyo 113, Japan. 
Although our model is simple and suitable for physical discussion, it has three important limitations. The annulus approximation is justified only when the flow pattern is roll convection with the axis parallel to the rotation vector. This situation is realized when the rotation effect is important and when the temperature heterogeneity on the boundary is sectorial. The first limitation is consequently that our model permits only sectorial temperature heterogeneity on the boundary. It does not, however, prevent us from applying our model to the Earth, because the predominance of sectorial degree two pattern in seismic heterogeneities in the lower mantle (e.g. DziEWONSKI, 1984; TANIMOTO, 1990; SU and DzIEWonski, 1991) signifies the same prevalent sectorial degree two pattern in the CMB thermal heterogeneity. Furthermore, it allows us to compare ZHANG and GuBBINS (1992)'s model with ours because their calculation is limited to sectorial temperature heterogeneities on the CMB. The second limitation is that the vertical stratification in the outer core is assumed to be neutral. This neutrality insures the predominance of the Coriolis effect, because strongly stable stratification would tend to confine the flow in a shallow part of the outer core (e.g. ZHANG and GuBbins, 1992). There is no reliable evidence for stable stratification in the Earth's outer core (MELCHIOR and DUCARME, 1986; ZÜRN et al., 1987; MAnsinha et al., 1990). The third limitation is the omission of the basic flow. This assumption is made for simplicity, because our main purpose is to obtain physically clear understanding. Besides, we have little information about the flow within the outer core. Effects of advection terms are discussed in more detail in Section 2.

Although this model may seem to have too many limitations, it is useful as a basis for considering the physical nature of the outer core due to its simplicity.

This paper is made up as follows. A linear analysis of the response of the outer core fluid to a CMB temperature heterogeneity is performed in Section 2. The result is applied to the Earth's core in Section 3. By comparing the spatial pattern of the geomagnetic field with that of the seismic heterogeneity in the lower mantle, we infer the strength of the toroidal field. In Section 4, we discuss the possibility of the dynamo action of the flow driven by a CMB temperature heterogeneity. Conclusions are given in Section 5 .

\section{Quasi-Geostrophic Model}

We consider the thermal response of the outer core fluid to the temperature heterogeneity on the CMB. As mentioned in Section 1, we assume that the pattern of the temperature distribution is sectorial, that no significant stable stratification exists in the outer core, and that the Coriolis force dominates in force balance. Under the assumptions above, a quasi-geostrophic model (e.g. Hide, 1966; Busse, 1970, 1975, 1976; Pedlosky, 1987) is useful in theoretical analyses.

The quasi-geostrophic model which is often used in studies of the outer core fluid is described. We consider a cylindrical annulus as shown in Fig. 1, which is supposed to represent the region outside the cylinder touching the inner core. Since the effect of rotation is assumed to be strong, the fluid motion tends to be uniform along the rotation axis (the Taylor-Proudman theorem), allowing us to employ the geometry of Fig. 1. The top and the bottom boundaries of the cylinder are inclined by the angle $\eta / 2$ to the equatorial plane to express the topographic $\beta$ effect (e.g. PEDLOSKY, 1987); vortex tubes parallel to the rotation vector acquire positive relative vorticity when they move toward the rotation axis and vice versa.

The gravity vector is assumed to point toward the rotation axis. Only this component of the gravity is important in the quasi-geostrophic model (YANO, 1992).

We use local cartesian coordinates $(x, y, z)$ with corresponding unit vectors $\mathbf{i}, \mathbf{j}, \mathbf{k}$ pointing in the radial, azimuthal and axial directions, respectively. 


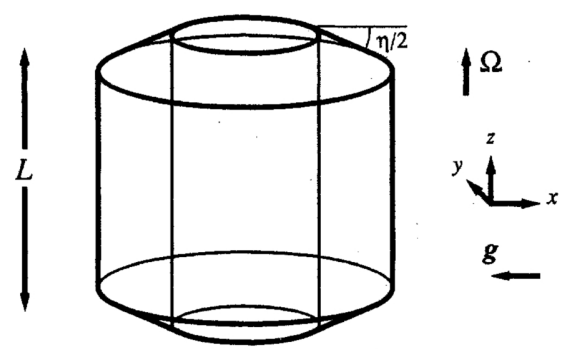

Fig. 1. Geometrical configuration used in the theoretical analysis. The outer core is modeled by the annulus with inclined top and bottom boundaries.

The basic equations are the linearized Boussinesq equations. They are given by

$$
\begin{aligned}
\left(\frac{\partial}{\partial t}-\nu \nabla^{2}\right) \mathbf{v}+2 \boldsymbol{\Omega} \times \mathbf{v} & =-\nabla \pi+\alpha g \theta \mathbf{i}+(\mathbf{B} \cdot \nabla) \mathbf{b}+(\mathbf{b} \cdot \nabla) \mathbf{B}, \\
\nabla \cdot \mathbf{v} & =0 \\
\left(\frac{\partial}{\partial t}-\lambda \nabla^{2}\right) \mathbf{b}+(\mathbf{v} \cdot \nabla) \mathbf{B} & =(\mathbf{B} \cdot \nabla) \mathbf{v}, \\
\nabla \cdot \mathbf{b} & =0, \\
\left(\frac{\partial}{\partial t}-\kappa \nabla^{2}\right) \theta & =0,
\end{aligned}
$$

where $\mathbf{v}$ is the flow velocity, $\mathbf{B}$ is the basic magnetic field, $\mathbf{b}$ is the linear part of the magnetic field, $\theta$ is the temperature, $\pi$ is the pressure divided by the density, $\nu$ is the kinematic viscosity, $\lambda$ the magnetic diffusivity, $\kappa$ is the thermal diffusivity, $\boldsymbol{\Omega}$ is the rotation vector, $\alpha$ is the coefficient of thermal expansion, and $g$ is the gravity. The magnetic field is measured in the Alfvèn velocity, which is the magnetic flux density divided by $\sqrt{\rho \mu}$ in the SI unit, where $\rho$ is the density, and $\mu$ is the magnetic permeability. The basic magnetic field is given by

$$
\mathbf{B}=V_{A}(\mathbf{i} F(z)+\mathbf{j} G(z)) .
$$

A few comments should be made about the choice of the basic field. First, we set the basic vertical temperature gradient to be zero as noted before, because no reliable evidence exists for stable stratification in the Earth's outer core. If the stratification were so stable that the Brunt-Väisälä frequency exceeds the rotation frequency, the hydrostatic approximation would be appropriate, and the present analysis based on the quasi-geostrophic approximation would fail. Second, we set the basic flow to be zero for simplicity. It is not practical to include the effect of the basic flow, because we have little information about the flow inside the outer core. Rather it is important to clarify the physical mechanism at this stage. Although this assumption makes the problem easy to solve and to understand, it may make the analysis inapplicable to the Earth's core. In the temperature equation, for example, the ratio of the advection term due to the basic field, which is omitted, to the diffusion term is given approximately by the Péclet number,

$$
P e=V L / \kappa,
$$

where $V$ is the typical magnitude of the basic flow velocity. In the Earth's core, $\kappa$, which is interpreted as the eddy diffusivity, is not known, but its upper bound is probably given by $V L$, as is the case for the mixing length theory with $V$ being the scale of the velocity fluctuation, 
and $L$ being the mixing length. Thus $P e$ is at least of the order of unity. If $P e$ is of the order of unity, our analysis is marginally appropriate for applying to the Earth's core. If $P e$ exceeds the order of unity, our analysis fails. It should be noted, however, that there are practically no means to calculate the flow with $P e \gg 1$. Third, we include only the $z$-dependence in the basic magnetic field for simplicity. In the Earth's core $x$-dependence of $G$ would exist, because the toroidal magnetic field is zero at the $\mathrm{CMB}$, and increases toward the interior.

The boundary conditions for the velocity are

$$
\begin{array}{llll}
v_{x}=0 & \text { at } & x=-D, 0 \\
v_{z}=\mp \frac{1}{2} \eta v_{x} & \text { at } & z= \pm \frac{1}{2} L
\end{array}
$$

and

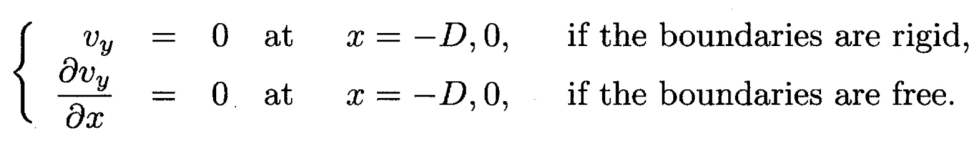

If we assume that the mantle is insulating, the boundary condition for the magnetic field becomes

$$
\mathbf{b}=\mathbf{b}_{\text {ext }} \quad \text { at the boundaries, }
$$

where $\mathbf{b}_{\text {ext }}$ is a potential field outside the annulus. The boundary conditions for the temperature are

$$
\begin{array}{llll}
\theta=\Delta T e^{i l y} & \text { at } & x=0, \\
\theta=0 & \text { at } & x=-D .
\end{array}
$$

From now on, we set $D \rightarrow \infty$ for simplicity, and get stationary solutions.

Equation (5) can be solved easily.

$$
\theta=\Delta T e^{i l y+l x}
$$

We decompose $\mathbf{v}$ and $\mathbf{b}$ in the following way so that the solenoidal conditions (2) and (4) are satisfied.

$$
\begin{aligned}
& \mathbf{v}=\nabla \times \mathbf{k} \psi+\nabla \times \nabla \times \mathbf{k} \phi, \\
& \mathbf{b}=V_{A}(\nabla \times \mathbf{k} g+\nabla \times \nabla \times \mathbf{k} h) .
\end{aligned}
$$

Substituting (13) and (14) into the $z$ component of the curl curl of Eq. (1), we obtain,

$$
\begin{aligned}
2 \Omega \frac{\partial}{\partial z} \triangle_{2} \psi= & -\left(\frac{\partial}{\partial t}-\nu \nabla^{2}\right) \nabla^{2} \triangle_{2} \phi+\alpha g \frac{\partial^{2} \theta}{\partial x \partial z} \\
& +V_{A}^{2}\left[\left(F \frac{\partial}{\partial x}+G \frac{\partial}{\partial y}\right) \nabla^{2} \triangle_{2} h-\left(\frac{d^{2} F}{d z^{2}} \frac{\partial}{\partial x}+\frac{d^{2} G}{d z^{2}} \frac{\partial}{\partial y}\right) \triangle_{2} h\right]
\end{aligned}
$$

where

$$
\triangle_{2}=\frac{\partial^{2}}{\partial x^{2}}+\frac{\partial^{2}}{\partial y^{2}}
$$

The $z$ component of the curl of Eq. (1) becomes

$$
\begin{aligned}
2 \Omega \frac{\partial}{\partial z} \triangle_{2} \phi= & \left(\frac{\partial}{\partial t}-\nu \nabla^{2}\right) \triangle_{2} \psi-\alpha g \frac{\partial \theta}{\partial y} \\
& +V_{A}^{2}\left[-\left(F \frac{\partial}{\partial x}+G \frac{\partial}{\partial y}\right) \triangle_{2} g+\left(\frac{d F}{d z} \frac{\partial}{\partial y}-\frac{d G}{d z} \frac{\partial}{\partial x}\right) \triangle_{2} h\right] .
\end{aligned}
$$


The $z$ component of the curl of Eq. (3) gives

$$
\left(\frac{\partial}{\partial t}-\lambda \nabla^{2}\right) \triangle_{2} g=\left(F \frac{\partial}{\partial x}+G \frac{\partial}{\partial y}\right) \triangle_{2} \psi+\left(\frac{d F}{d z} \frac{\partial}{\partial y}-\frac{d G}{d z} \frac{\partial}{\partial x}\right) \triangle_{2} \phi
$$

The $z$ component of Eq. (3) becomes

$$
\left(\frac{\partial}{\partial t}-\lambda \nabla^{2}\right) \triangle_{2} h=\left(F \frac{\partial}{\partial x}+G \frac{\partial}{\partial y}\right) \triangle_{2} \phi
$$

The boundary conditions for the velocity (8) and (9) become

$$
\begin{aligned}
& \frac{\partial \psi}{\partial y}+\frac{\partial^{2} \phi}{\partial x \partial y}=0 \quad \text { at } x=-\infty, 0 \\
& \triangle_{2} \phi= \pm \frac{1}{2} \eta\left(\frac{\partial \psi}{\partial y}+\frac{\partial^{2} \phi}{\partial x \partial z}\right) \quad \text { at } \quad z= \pm \frac{1}{2} L,
\end{aligned}
$$

and

$$
\left\{\begin{aligned}
-\frac{\partial \psi}{\partial x}+\frac{\partial^{2} \phi}{\partial y \partial z}=0 \quad \text { at } \quad x=-\infty, 0, & \text { if the boundaries are rigid } \\
\frac{\partial}{\partial x}\left(-\frac{\partial \psi}{\partial x}+\frac{\partial^{2} \phi}{\partial y \partial z}\right)=0 \quad \text { ar } & x=-\infty, 0, \quad \text { if the boundaries are free. }
\end{aligned}\right.
$$

Now the problem is reduced to solving equations (15), (17), (18), and (19) with $\theta$ given by (12). We shall go on to derive quasi-geostrophic equations, using a scale analysis under the assumption that the Coriolis force is large. We introduce non-dimensional variables denoted by ( a circumflex).

$$
\begin{aligned}
t & =\frac{L^{2}}{\lambda} \hat{t}, \\
x & =L \hat{x} \\
y & =L \hat{y} \\
z & =L \hat{z} \\
l & =L^{-1} \hat{l} \\
\psi & =\frac{\alpha g \Delta T}{\beta} \hat{\psi}, \\
\phi & =\frac{\alpha g \Delta T L}{\beta} \hat{\phi}, \\
g & =\frac{\alpha g \Delta T L}{\beta \lambda} \hat{g}, \\
h & =\frac{\alpha g \Delta T L^{2}}{\beta \lambda} \hat{h}, \\
\theta & =\Delta T \hat{\theta},
\end{aligned}
$$

where $\beta$ represents the topographic $\beta$ effect.

$$
\beta=2 \Omega \frac{\eta}{L}
$$


Equations (12), (15), (17), (18), and (19) may be rewritten as follows.

$$
\begin{aligned}
& \frac{\partial}{\partial \hat{z}} \hat{\triangle}_{2} \hat{\psi}=-\epsilon_{D}\left(\frac{\partial}{\partial \hat{t}}-P_{m} \hat{\nabla}^{2}\right) \hat{\nabla}^{2} \hat{\triangle}_{2} \hat{\phi}+\eta \frac{\partial^{2} \hat{\theta}}{\partial \hat{x} \partial \hat{z}} \\
&+\epsilon_{E}\left[\left(F \frac{\partial}{\partial \hat{x}}+G \frac{\partial}{\partial \hat{y}}\right) \hat{\nabla}^{2} \hat{\triangle}_{2} \hat{h}-\left(\frac{d^{2} F}{d \hat{z}^{2}} \frac{\partial}{\partial \hat{x}}+\frac{d^{2} G}{d \hat{z}^{2}} \frac{\partial}{\partial \hat{y}}\right) \hat{\triangle}_{2} \hat{h}\right] \\
& \frac{\partial}{\partial \hat{z}} \hat{\triangle}_{2} \hat{\phi}= \epsilon_{D}\left(\frac{\partial}{\partial \hat{t}}-P_{m} \hat{\nabla}^{2}\right) \hat{\triangle}_{2} \hat{\psi}-\eta \frac{\partial \hat{\theta}}{\partial \hat{y}} \\
&+\epsilon_{E}\left[-\left(F \frac{\partial}{\partial \hat{x}}+G \frac{\partial}{\partial \hat{y}}\right) \hat{\triangle}_{2} \hat{g}+\left(\frac{d F}{d \hat{z}} \frac{\partial}{\partial \hat{y}}-\frac{d G}{d \hat{z}} \frac{\partial}{\partial \hat{x}}\right) \hat{\triangle}_{2} \hat{h}\right] \\
&\left(\frac{\partial}{\partial \hat{t}}-\hat{\nabla}^{2}\right) \hat{\triangle}_{2} \hat{g}=\left(F \frac{\partial}{\partial \hat{x}}+G \frac{\partial}{\partial \hat{y}}\right) \hat{\triangle}_{2} \hat{\psi}+\left(\frac{d F}{d \hat{z}} \frac{\partial}{\partial \hat{y}}-\frac{d G}{d \hat{z}} \frac{\partial}{\partial \hat{x}}\right) \hat{\triangle}_{2} \hat{\phi} \\
&\left(\frac{\partial}{\partial \hat{t}}-\hat{\nabla}^{2}\right) \hat{\triangle}_{2} \hat{h}=\left(F \frac{\partial}{\partial \hat{x}}+G \frac{\partial}{\partial \hat{y}}\right) \hat{\triangle}_{2} \hat{\phi}
\end{aligned}
$$

with

$$
\hat{\theta}=e^{i \hat{l} \hat{y}+\hat{l} \hat{x}}
$$

Here

$$
\begin{aligned}
\epsilon_{D} & =\frac{\lambda}{2 \Omega L^{2}}, \\
\epsilon_{E} & =\frac{V_{A}^{2}}{2 \Omega \lambda},
\end{aligned}
$$

and $P_{m}$ is the magnetic Prandtl number.

$$
P_{m}=\frac{\nu}{\lambda}
$$

The boundary conditions for the velocity (20) and (21) may be rewritten as

$$
\begin{aligned}
& \frac{\partial \hat{\psi}}{\partial \hat{y}}+\frac{\partial^{2} \hat{\phi}}{\partial \hat{x} \partial \hat{y}}=0 \quad \text { at } \hat{x}=-\infty, 0 \\
& \hat{\triangle}_{2} \hat{\phi}= \pm \frac{1}{2} \eta\left(\frac{\partial \hat{\psi}}{\partial \hat{y}}+\frac{\partial^{2} \hat{\phi}}{\partial \hat{x} \partial \hat{z}}\right) \text { at } \hat{z}= \pm \frac{1}{2},
\end{aligned}
$$

and

$$
\left\{\begin{aligned}
&-\frac{\partial \hat{\psi}}{\partial \hat{x}}+\frac{\partial^{2} \hat{\phi}}{\partial \hat{y} \partial \hat{z}}=0 \quad \text { at } \quad \hat{x}=-\infty, 0, \text { if the boundaries are rigid } \\
& \frac{\partial}{\partial \hat{x}}\left(-\frac{\partial \hat{\psi}}{\partial \hat{x}}+\frac{\partial^{2} \hat{\phi}}{\partial \hat{y} \partial \hat{z}}\right)=0 \quad \text { at } \quad \hat{x}=-\infty, 0, \quad \text { if the boundaries are free. }
\end{aligned}\right.
$$

Assuming that

$$
\eta \sim \epsilon_{D} \sim \epsilon_{E} \ll 1
$$

and

$$
P_{m}=O(1)
$$


we expand variables in terms of $\eta$.

$$
\begin{aligned}
\hat{\psi} & =\hat{\psi}_{0}+\eta \hat{\psi}_{1}+\eta^{2} \hat{\psi}_{2}+\cdots, \\
\hat{\phi} & =\hat{\phi}_{0}+\eta \hat{\phi}_{1}+\eta^{2} \hat{\phi}_{2}+\cdots, \\
\hat{g} & =\hat{g}_{0}+\eta \hat{g}_{1}+\eta^{2} \hat{g}_{2}+\cdots \\
\hat{h} & =\hat{h}_{0}+\eta \hat{h}_{1}+\eta^{2} \hat{h}_{2}+\cdots
\end{aligned}
$$

The zeroth order parts of Eqs. (33), (34), (35), and (36) are

$$
\begin{aligned}
\frac{\partial}{\partial \hat{z}} \hat{\triangle}_{2} \hat{\psi}_{0} & =0 \\
\frac{\partial}{\partial \hat{z}} \hat{\triangle}_{2} \hat{\phi}_{0} & =0 \\
\left(\frac{\partial}{\partial \hat{t}}-\hat{\nabla}^{2}\right) \hat{\triangle}_{2} \hat{g}_{0} & =\left(F \frac{\partial}{\partial \hat{x}}+G \frac{\partial}{\partial \hat{y}}\right) \hat{\triangle}_{2} \hat{\psi}_{0}+\left(\frac{d F}{d \hat{z}} \frac{\partial}{\partial \hat{y}}-\frac{d G}{d \hat{z}} \frac{\partial}{\partial \hat{x}}\right) \hat{\triangle}_{2} \hat{\phi}_{0} \\
\left(\frac{\partial}{\partial \hat{t}}-\hat{\nabla}^{2}\right) \hat{\triangle}_{2} \hat{h}_{0} & =\left(F \frac{\partial}{\partial \hat{x}}+G \frac{\partial}{\partial \hat{y}}\right) \hat{\triangle}_{2} \hat{\phi}_{0} .
\end{aligned}
$$

The boundary conditions are

$$
\begin{aligned}
\frac{\partial \hat{\psi}_{0}}{\partial \hat{y}}+\frac{\partial^{2} \hat{\phi}_{0}}{\partial \hat{x} \partial \hat{y}} & =0 \text { at } & \hat{x}=-\infty, 0 \\
\hat{\triangle}_{2} \hat{\phi}_{0} & =0 \quad \text { at } & \hat{z}= \pm \frac{1}{2}
\end{aligned}
$$

The solution for $\hat{\psi}_{0}$ is given by

$$
\hat{\psi}_{0}=\hat{\psi}_{0}(\hat{x}, \hat{y}, \hat{t})
$$

where

$$
\begin{aligned}
\hat{\psi}_{0}(0, \hat{y}, \hat{t}) & =0 \\
\hat{\psi}_{0}(-\infty, \hat{y}, \hat{t}) & =0 .
\end{aligned}
$$

This corresponds to the Taylor-Proudman theorem, which shows that a steady flow is twodimensional (i.e. independent of $z$ ) when the Coriolis force is large. The variables $\hat{\phi}_{0}$ and $\hat{h}_{0}$ are solved to be 0 , and $\hat{g}_{0}$ is given as the solution of

$$
\left(\frac{\partial}{\partial \hat{t}}-\hat{\nabla}^{2}\right) \hat{g}_{0}=\left(F \frac{\partial}{\partial \hat{x}}+G \frac{\partial}{\partial \hat{y}}\right) \hat{\psi}_{0} .
$$

The boundary condition for $\hat{g}_{0}$ at $\hat{x}=0$ is given by

$$
\frac{\partial \hat{g}_{0} / \partial \hat{x}}{\partial \hat{g}_{0} / \partial \hat{y}}=i
$$

provided that $\hat{g}_{0}$ is weakly dependent on $z$, because $\hat{g}_{\text {ext }, 0}$ outside the boundary is then

$$
\hat{g}_{e x t, 0} \propto e^{i \hat{\imath} \hat{y}} e^{-\hat{l} \hat{x}} .
$$

The boundary condition for $\hat{g}_{0}$ for $\hat{x} \rightarrow-\infty$ is given by

$$
\hat{g}_{0} \rightarrow 0 \text {. }
$$


Let us proceed to the first order of Eq. (34).

$$
\frac{\partial}{\partial \hat{z}} \hat{\triangle}_{2} \hat{\phi}_{1}=\frac{\epsilon_{D}}{\eta}\left(\frac{\partial}{\partial \hat{t}}-P_{m} \hat{\triangle}_{2}\right) \hat{\triangle}_{2} \hat{\psi}_{0}-\frac{\partial \hat{\theta}}{\partial \hat{y}}-\frac{\epsilon_{E}}{\eta}\left(F \frac{\partial}{\partial \hat{x}}+G \frac{\partial}{\partial \hat{y}}\right) \hat{\triangle}_{2} \hat{g}_{0} .
$$

The boundary condition is

$$
\hat{\triangle}_{2} \hat{\phi}_{1}= \pm \frac{1}{2} \frac{\partial \hat{\psi}_{0}}{\partial \hat{y}} \quad \text { at } \quad \hat{z}= \pm \frac{1}{2} .
$$

The solvability condition is obtained by integrating equation (61) from $\hat{z}=-1 / 2$ to $\hat{z}=1 / 2$ and applying the boundary condition (62), giving

$$
\left[-\eta \frac{\partial}{\partial \hat{y}}+\epsilon_{D}\left(\frac{\partial}{\partial \hat{t}}-P_{m} \hat{\triangle}_{2}\right) \hat{\triangle}_{2}\right] \hat{\psi}_{0}-\epsilon_{E} \int_{-1 / 2}^{1 / 2}\left(F \frac{\partial}{\partial \hat{x}}+G \frac{\partial}{\partial \hat{y}}\right) \hat{\triangle}_{2} \hat{g}_{0} d \hat{z}=\eta \frac{\partial \hat{\theta}}{\partial \hat{y}} .
$$

We substitute (57) into (63) to obtain

$$
\begin{aligned}
& \left\{\left(\frac{\partial}{\partial \hat{t}}-\hat{\triangle}_{2}\right)\left[-\eta \frac{\partial}{\partial \hat{y}}+\epsilon_{D}\left(\frac{\partial}{\partial \hat{t}}-P_{m} \hat{\triangle}_{2}\right) \hat{\triangle}_{2}\right]\right. \\
& \left.-\epsilon_{E}\left(\left\langle F^{2}\right\rangle \frac{\partial^{2}}{\partial \hat{x}^{2}}+2\langle F G\rangle \frac{\partial^{2}}{\partial \hat{x} \partial \hat{y}}+\left\langle G^{2}\right\rangle \frac{\partial^{2}}{\partial \hat{y}^{2}}\right) \hat{\triangle}_{2}\right\} \hat{\psi}_{0} \\
& =\eta\left(\frac{\partial}{\partial \hat{t}}-\hat{\triangle}_{2}\right) \frac{\partial \hat{\theta}}{\partial \hat{y}}
\end{aligned}
$$

where

$$
\langle\cdot\rangle=\int_{-1 / 2}^{1 / 2}(\cdot) d \hat{z}=\frac{1}{L} \int_{-L / 2}^{L / 2}(\cdot) d z
$$

is a vertical mean. This is a quasi-geostrophic equation. We shall consider only stationary solutions, in which case the equation reduces to

$$
\left\{\left[\eta \frac{\partial}{\partial \hat{y}}+\epsilon_{D} P_{m} \hat{\triangle}_{2}^{2}\right]-\epsilon_{E}\left(\left\langle F^{2}\right\rangle \frac{\partial^{2}}{\partial \hat{x}^{2}}+2\langle F G\rangle \frac{\partial^{2}}{\partial \hat{x} \partial \hat{y}}+\left\langle G^{2}\right\rangle \frac{\partial^{2}}{\partial \hat{y}^{2}}\right)\right\} \hat{\psi}_{0}=-\eta \frac{\partial \hat{\theta}}{\partial \hat{y}}
$$

To summarize, we shall solve the following equations in a dimensional form. The equation for $\psi_{0}$ is

$$
\left\{\left[\beta \frac{\partial}{\partial y}+\nu \triangle_{2}^{2}\right]-\frac{V_{A}^{2}}{\lambda}\left(\left\langle F^{2}\right\rangle \frac{\partial^{2}}{\partial x^{2}}+2\langle F G\rangle \frac{\partial^{2}}{\partial x \partial y}+\left\langle G^{2}\right\rangle \frac{\partial^{2}}{\partial y^{2}}\right)\right\} \psi_{0}=-\alpha g \frac{\partial \theta}{\partial y}
$$

with the boundary conditions

$$
\begin{aligned}
\psi_{0}(0, y, t) & =0 \\
\psi_{0}(-\infty, y, t) & =0
\end{aligned}
$$

The equation for $g_{0}$ is

$$
\left(\frac{\partial}{\partial t}-\lambda \triangle_{2}\right) g_{0}=\left(F \frac{\partial}{\partial x}+G \frac{\partial}{\partial y}\right) \psi_{0}
$$

with the boundary condition

$$
\begin{aligned}
\frac{\partial g_{0} / \partial x}{\partial g_{0} / \partial y} & =i \text { at } \quad x=0 \\
g_{0} & \rightarrow 0 \text { for } \quad x \rightarrow-\infty
\end{aligned}
$$


if $g_{0}$ is weakly dependent on $z$. The variables $\phi_{0}$ and $h_{0}$ are 0 .

We assume from now on that

$$
E \ll 1,
$$

which is usually considered to be valid in the outer core. Here,

$$
E \equiv \frac{\nu l^{3}}{\beta}
$$

is the Ekman number defined in terms of $\beta$. The Elsasser number $\mathcal{E}$ defined in terms of $\beta$ is introduced as a measure of the strength of the magnetic field.

$$
\mathcal{E} \equiv \frac{V_{A}^{2} l\left\langle G^{2}\right\rangle}{\beta \lambda}
$$

We assume that the poloidal field is weaker than the toroidal field.

$$
\mathcal{P} \equiv \frac{\left\langle F^{2}\right\rangle}{\left\langle G^{2}\right\rangle} \ll 1
$$

We shall investigate the following two cases:

- when viscosity can be neglected

- when the poloidal magnetic field can be neglected

\subsection{Response of an inviscid fluid}

We consider the response of an inviscid core to the temperature heterogeneity on the coremantle boundary. The neglect of the viscous term is justified in the following two circumstances:

- $\mathcal{E} \ll 1$ and

$$
(\mathcal{E P})^{2} E^{-1} \gg 1
$$

- $\mathcal{E} \gg 1$ and

$$
\mathcal{E} \mathcal{P}^{2} E^{-1} \gg 1
$$

The basic equation is obtained from (67) by setting $\nu=0$.

$$
\left[\beta \frac{\partial}{\partial y}-\frac{V_{A}^{2}}{\lambda}\left(\left\langle F^{2}\right\rangle \frac{\partial^{2}}{\partial x^{2}}+2\langle F G\rangle \frac{\partial^{2}}{\partial x \partial y}+\left\langle G^{2}\right\rangle \frac{\partial^{2}}{\partial y^{2}}\right)\right] \psi_{0}=-\alpha g \frac{\partial \theta}{\partial y}
$$

where

$$
\theta=\Delta T e^{i l y+l x} .
$$

The boundary conditions are given by Eqs. (68) and (69).

We obtain the solution for $\psi_{0}$.

$$
\psi_{0}=-\frac{\alpha g \Delta T}{\beta} \frac{1}{1+i \mathcal{E}(\mathcal{P}+2 i \mathcal{Q}-1)} e^{i l y}\left(e^{l x}-e^{\left(k_{1}+i k_{2}\right) x}\right),
$$

where

$$
\mathcal{Q} \equiv \frac{\langle F G\rangle}{\left\langle G^{2}\right\rangle}
$$

From the Schwarz's inequality,

$$
\mathcal{Q}^{2} \leq \mathcal{P} \ll 1
$$


The wave numbers $k_{1}$ and $k_{2}$ are given by

$$
\begin{aligned}
\frac{k_{1}}{l} & =\frac{1}{\mathcal{P}} \sqrt{\frac{1}{2}\left[\mathcal{P}-\mathcal{Q}^{2}+\sqrt{\left(\mathcal{P}-\mathcal{Q}^{2}\right)^{2}+\left(\mathcal{P} \mathcal{E}^{-1}\right)^{2}}\right]} \\
\frac{k_{2}}{l} & =\frac{1}{\mathcal{P}}\left\{-\mathcal{Q}+\sqrt{\frac{1}{2}\left[-\left(\mathcal{P}-\mathcal{Q}^{2}\right)+\sqrt{\left(\mathcal{P}-\mathcal{Q}^{2}\right)^{2}+\left(\mathcal{P} \mathcal{E}^{-1}\right)^{2}}\right]} .\right.
\end{aligned}
$$

If $\mathcal{E} \ll 1$,

$$
\begin{aligned}
& \frac{k_{1}}{l} \simeq \sqrt{\frac{1}{2 \mathcal{E P}}} \gg 1, \\
& \frac{k_{2}}{l} \simeq \sqrt{\frac{1}{2 \mathcal{E P}}} \gg 1 .
\end{aligned}
$$

Since $k_{1} \gg l$, a thin boundary layer is formed. If $\mathcal{E} \gg 1$,

$$
\begin{aligned}
\frac{k_{1}}{l} & \simeq \sqrt{\frac{1}{\mathcal{P}}\left(1-\frac{\mathcal{Q}^{2}}{\mathcal{P}}\right)}, \\
\frac{k_{2}}{l} & \simeq-\frac{\mathcal{Q}}{\mathcal{P}}+\frac{1}{2 \mathcal{E}} \sqrt{\frac{1}{\mathcal{P}-\mathcal{Q}^{2}}} .
\end{aligned}
$$

If $\mathcal{Q}^{2} \ll \mathcal{P}$ in (87), then $k_{1} \gg l$; a thin boundary layer is formed.

Now consider the location of upwellings and downwellings. Equation (80) may be rewritten as follows:

$$
\psi_{0}=-\frac{\alpha g \Delta T}{\beta} \frac{1}{\sqrt{(1-2 \mathcal{E} \mathcal{Q})^{2}+\mathcal{E}^{2}(1-\mathcal{P})^{2}}} e^{i(l y+\delta)}\left(e^{l x}-e^{\left(k_{1}+i k_{2}\right) x}\right)
$$

where

$$
\delta=\left\{\begin{array}{lll}
\arctan \frac{\mathcal{E}(1-\mathcal{P})}{1-2 \mathcal{E Q}} & \text { if } & 1-2 \mathcal{E Q}>0 \\
\arctan \frac{\mathcal{E}(1-\mathcal{P})}{1-2 \mathcal{E Q}}+\pi & \text { if } & 1-2 \mathcal{E} \mathcal{Q}<0 .
\end{array}\right.
$$

$\delta$ represents the location of upwellings and downwellings in the interior region (outside the boundary layer). If $\mathcal{E} \ll 1, \delta \simeq 0$; upwellings occur to the east of hot regions and downwellings occur to the west of hot regions as ZHANG and GubBINS (1992) calculated. This is caused by the balance between the topographic $\beta$ effect and the buoyancy force.

$$
\beta \frac{\partial \psi_{0}}{\partial y}=-\alpha g \frac{\partial \theta}{\partial y} .
$$

This situation is illustrated in Fig. 2(a). If $\mathcal{E} \gg 1, \delta \simeq \pi / 2$; upwellings occur beneath hot regions, and downwellings occur beneath cold regions as BLOxHAM and GUBBINs (1987) suggested. This is caused by the balance between the Lorentz force and the buoyancy force.

$$
-\frac{V_{A}^{2}}{\lambda}\left\langle G^{2}\right\rangle \frac{\partial^{2} \psi_{0}}{\partial y^{2}}=-\alpha g \frac{\partial \theta}{\partial y}
$$

This situation is illustrated in Fig. 2(b). 
(a)

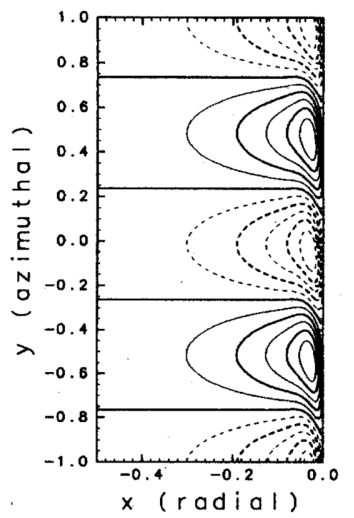

(b)

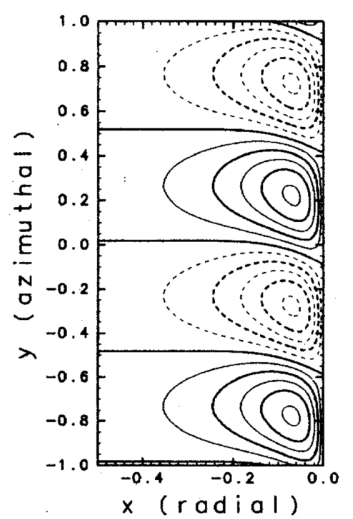

Fig. 2. Contours of the stream function of the inviscid flow induced by thermal heterogeneity on the CMB in the presence of the magnetic field. The abscissa is radial direction outward to the right. The ordinate represents azimuthal direction. Flow along solid contours are cyclonic rolls (i.e. counterclockwise rotation). Flow along dashed contours are anticyclonic rolls (i.e. clockwise rotation). Temperature distribution is given as $\theta=\Delta T \cos 2 \pi y$ at $x=0$. The unit of the amplitude is $\alpha g \Delta T / \beta$. (a). For $\mathcal{E}=0.1, \mathcal{P}=0.1, \mathcal{Q}=0$. The contour interval is 0.15 . (b). For $\mathcal{E}=10, \mathcal{P}=0.1, \mathcal{Q}=0$. The contour interval is 0.012 .

The magnetic field can be calculated from Eq. (70) with the boundary conditions (71), when its dependence on $z$ is weak. The solution within the outer core is

$$
\begin{aligned}
g_{0}= & \frac{\alpha g \Delta T}{\lambda \beta} \frac{1}{1+i \mathcal{E}(\mathcal{P}+2 i \mathcal{Q}-1)} e^{i l y}\left[(F+i G)\left(\frac{x}{2}-\frac{1}{4 l}\right) e^{l x}\right. \\
& \left.+\frac{F\left(k_{1}+i k_{2}\right)+i G l}{\left(k_{1}+i k_{2}\right)^{2}-l^{2}}\left(e^{\left(k_{1}+i k_{2}\right) x}-\frac{k_{1}+i k_{2}+l}{2 l} e^{l x}\right)\right]
\end{aligned}
$$

and that within the mantle is

$$
\begin{aligned}
g_{e x t, 0}= & -\frac{\alpha g \Delta T}{\lambda \beta} \frac{1}{1+i \mathcal{E}(\mathcal{P}+2 i \mathcal{Q}-1)} \\
& \times\left[\frac{F+i G}{4 l}+\frac{F\left(k_{1}+i k_{2}\right)+i G l}{\left(k_{1}+i k_{2}\right)^{2}-l^{2}} \frac{k_{1}+i k_{2}-l}{2 l}\right] e^{i l y-l x} .
\end{aligned}
$$

The helicity is an important variable to dynamo problems.

$$
W=\overline{\mathbf{v} \cdot \nabla \times \mathbf{v}},
$$

where $\overline{(\cdot)}$ represents an average. Assuming that $\partial / \partial z \simeq 0$, we rewrite the helicity to the first order of $\eta$ by

$$
W_{1}(z)=\frac{1}{4}\left(\overline{{\triangle_{2} \phi_{1}^{\dagger} \triangle_{2} \psi_{0}}}-\overline{\frac{\partial \psi_{0}^{\dagger}}{\partial x} \frac{\partial}{\partial x} \Delta_{2} \phi_{1}}-\overline{\frac{\partial \psi_{0}^{\dagger}}{\partial y} \frac{\partial}{\partial y} \Delta_{2} \phi_{1}}\right)+\text { c.c. },
$$

where $\overline{(\cdot \quad)}$ represents horizontal average.

We calculate $\phi$ in order to obtain the helicity. We have seen that $\phi_{0}=0$. We obtain $\phi_{1}$ from Eq. (61),

$$
2 \Omega \frac{\partial}{\partial z} \triangle_{2} \phi_{1}=\frac{V_{A}^{2}}{\lambda}\left(F \frac{\partial}{\partial x}+G \frac{\partial}{\partial y}\right)^{2} \psi_{0}-\alpha g \frac{\partial \theta}{\partial y} .
$$


From Eqs. (78) and (97) we obtain

$$
\begin{aligned}
2 \Omega \frac{\partial}{\partial z} \triangle_{2} \phi_{1}= & \left\{\beta \frac{\partial}{\partial y}+\frac{V_{A}^{2}}{\lambda}\left[\left(F^{2}-\left\langle F^{2}\right\rangle\right) \frac{\partial^{2}}{\partial x^{2}}\right.\right. \\
& \left.\left.+2(F G-\langle F G\rangle) \frac{\partial^{2}}{\partial x \partial y}+\left(G^{2}-\left\langle G^{2}\right\rangle\right) \frac{\partial^{2}}{\partial y^{2}}\right]\right\} \psi_{0} .
\end{aligned}
$$

Integration in the $z$ direction gives

$$
\triangle_{2} \phi_{1}=\left[\frac{\beta}{2 \Omega} z \frac{\partial}{\partial y}+\frac{V_{A}^{2}\left\langle G^{2}\right\rangle L}{2 \Omega \lambda}\left(f_{F F}(z) \frac{\partial^{2}}{\partial x^{2}}+2 f_{F G}(z) \frac{\partial^{2}}{\partial x \partial y}+f_{G G}(z) \frac{\partial^{2}}{\partial y^{2}}\right)\right] \psi_{0},
$$

where

$$
\begin{aligned}
f_{F F}(z) & =\frac{1}{L} \int_{-L / 2}^{z} \frac{F^{2}}{\left\langle G^{2}\right\rangle} d z-\left(\frac{z}{L}+\frac{1}{2}\right) \mathcal{P} \\
f_{F G}(z) & =\frac{1}{L} \int_{-L / 2}^{z} \frac{F G}{\left\langle G^{2}\right\rangle} d z-\left(\frac{z}{L}+\frac{1}{2}\right) \mathcal{Q}, \\
f_{G G}(z) & =\frac{1}{L} \int_{-L / 2}^{z} \frac{G^{2}}{\left\langle G^{2}\right\rangle} d z-\left(\frac{z}{L}+\frac{1}{2}\right) .
\end{aligned}
$$

The helicity is obtained by substituting (80) and (99) into Eq. (96). In calculating the helicity, we assumed for simplicity that

$$
\overline{\left(e^{i \alpha x}\right)^{\dagger} e^{i \beta x} f(x)}=0
$$

if $\alpha \neq \beta$. Here $f(x)$ is an arbitrary real function of $x$. The helicity is

$$
\begin{aligned}
W_{1}(z)= & -\frac{(\alpha g \Delta T)^{2}}{2 \Omega \beta} \frac{1}{(1-2 \mathcal{E} \mathcal{Q})^{2}+\mathcal{E}^{2}(1-\mathcal{P})^{2}} \\
& \times\left\{e^{2 l x} l^{3} L \mathcal{E}\left(f_{F F}(z)-f_{G G}(z)\right)\right. \\
& +e^{2 k_{1} x}\left[l L \mathcal{E}\left(\left(k_{1}^{2}-k_{2}^{2}\right) f_{F F}(z)-2 l k_{2} f_{F G}(z)-l^{2} f_{G G}(z)\right)\right. \\
& \left.\left.-k_{1} k_{2}\left(z l+\frac{2 k_{1} L \mathcal{E}}{l}\left(k_{2} f_{F F}(z)+l f_{F G}(z)\right)\right)\right]\right\}
\end{aligned}
$$

As far as order estimation is concerned, the helicity in the interior region (i.e. outside the boundary layer) may symbolically be rewritten as

$$
W \sim \frac{(\alpha g \Delta T)^{2}}{2 \Omega \beta} l^{3} L \frac{\mathcal{E}}{1+\mathcal{E}^{2}},
$$

and the helicity in the boundary layer,

$$
W \sim \begin{cases}\frac{(\alpha g \Delta T)^{2}}{2 \Omega \beta} l^{3} L \frac{\mathcal{E}}{1+\mathcal{E}^{2}} & \text { if } \quad \mathcal{E P} \gg 1 \\ \frac{(\alpha g \Delta T)^{2}}{2 \Omega \beta} l^{3} L \frac{1}{2 \mathcal{E P}\left[1+\mathcal{E}^{2}\right]} & \text { if } \quad \mathcal{E P} \ll 1\end{cases}
$$




\subsection{Response of a viscid fluid without a poloidal magnetic field}

Next we consider the response of the viscid core without a poloidal magnetic field to the temperature heterogeneity on the core-mantle boundary. The neglect of the poloidal field is justified in the following two circumstances:

- $\mathcal{E} \ll 1$ and

$$
(\mathcal{E P})^{2} E^{-1} \ll 1
$$

- $\mathcal{E} \gg 1$ and

$$
\mathcal{E} \mathcal{P}^{2} E^{-1} \ll 1
$$

When the poloidal field is absent, the basic equation (67) becomes

$$
\left(\beta \frac{\partial}{\partial y}+\nu \triangle_{2}^{2}-\frac{V_{A}^{2}}{\lambda}\left\langle G^{2}\right\rangle \frac{\partial^{2}}{\partial y^{2}}\right) \psi_{0}=-\alpha g \frac{\partial \theta}{\partial y}
$$

where

$$
\theta=\Delta T e^{i l y+l x} .
$$

The boundary conditions are given by Eqs. (68), (69), and

$$
\frac{\partial^{2} \psi_{0}}{\partial x^{2}}(0, y, t)=0
$$

if the side boundaries are assumed to be free.

Using the assumption

$$
E \ll 1,
$$

we obtain the solution,

$$
\psi_{0}=-\frac{\alpha g \Delta T}{\beta} \frac{1}{1-i \mathcal{E}} e^{i l y}\left(e^{l x}-\frac{1}{2} e^{\left(k_{1}-i k_{2}\right) x}-\frac{1}{2} e^{\left(k_{2}+i k_{1}\right) x}\right)
$$

where

$$
\begin{aligned}
\frac{k_{1}}{l} & =\sqrt{\frac{1}{2}\left[\sqrt{\frac{1}{2}\left(-\mathcal{E}+\sqrt{1+\mathcal{E}^{2}}\right)}+\sqrt[4]{1+\mathcal{E}^{2}}\right]} E^{-1 / 4} \gg 1 \\
\frac{k_{2}}{l} & =\sqrt{\frac{1}{2}\left[-\sqrt{\frac{1}{2}\left(-\mathcal{E}+\sqrt{1+\mathcal{E}^{2}}\right)}+\sqrt[4]{1+\mathcal{E}^{2}}\right]} E^{-1 / 4} \gg 1 .
\end{aligned}
$$

To obtain the solution (113), we used the relation

$$
k_{1}, k_{2} \gg l \text {. }
$$

If $\mathcal{E} \ll 1$,

$$
\begin{aligned}
\frac{k_{1}}{l} & \simeq \sqrt{\frac{1+\sqrt{2}}{2 \sqrt{2}}} E^{-1 / 4} \gg 1, \\
\frac{k_{2}}{l} & \simeq \sqrt{\frac{-1+\sqrt{2}}{2 \sqrt{2}}} E^{-1 / 4} \gg 1 .
\end{aligned}
$$


If $\mathcal{E} \gg 1$,

$$
\begin{aligned}
\frac{k_{1}}{l} & \simeq \frac{1}{\sqrt{2}} \mathcal{E}^{1 / 4} E^{-1 / 4} \gg 1 \\
\frac{k_{2}}{l} & \simeq \frac{1}{\sqrt{2}} \mathcal{E}^{1 / 4} E^{-1 / 4} \gg 1 .
\end{aligned}
$$

Now we consider the location of upwellings and downwellings. Equation (113) may be rewritten as follows:

$$
\psi_{0}=-\frac{\alpha g \Delta T}{\beta} \frac{1}{\sqrt{1+\mathcal{E}^{2}}} e^{i(l y+\delta)}\left(e^{l x}-\frac{1}{2} e^{\left(k_{1}-i k_{2}\right) x}-\frac{1}{2} e^{\left(k_{2}+i k_{1}\right) x}\right),
$$

where

$$
\delta=\arctan \mathcal{E} .
$$

$\delta$ represents the location of upwellings and downwellings in the interior region (outside the boundary layer). If $\mathcal{E} \ll 1, \delta \simeq 0$; upwellings are located to the east of hot regions and downwellings to the west of hot regions as ZHANG and GuBBINS (1992) calculated. This is caused by the balance between the topographic $\beta$ effect and the buoyancy force.

$$
\beta \frac{\partial \psi_{0}}{\partial y}=-\alpha g \frac{\partial \theta}{\partial y} .
$$

This situation is illustrated in Fig. 3(a). If $\mathcal{E} \gg 1, \delta \simeq \pi / 2$; upwellings occur beneath hot regions, and downwellings occur beneath cold regions as BLOXHAM and GuBBINS (1987) suggested. This is caused by the balance between the Lorentz force and the buoyancy force.

$$
-\frac{V_{A}^{2}}{\lambda}\left\langle G^{2}\right\rangle \frac{\partial^{2} \psi_{0}}{\partial y^{2}}=-\alpha g \frac{\partial \theta}{\partial y}
$$

(a)

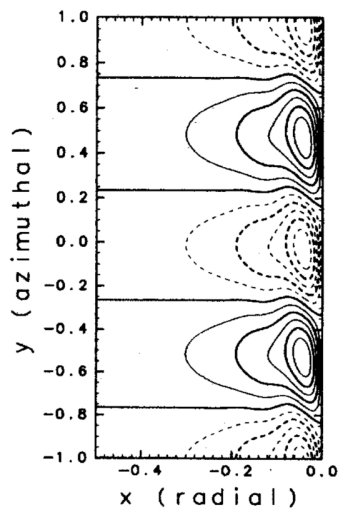

(b)

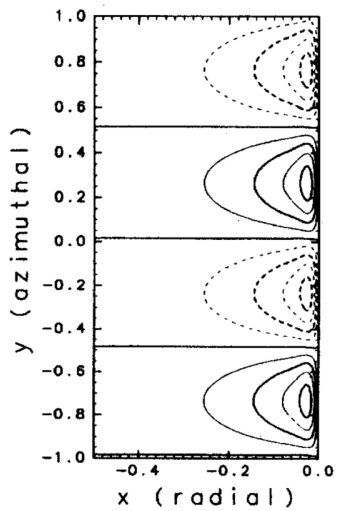

Fig. 3. Contours of the stream function of the flow induced by thermal heterogeneity on the CMB when the poloidal magnetic field is absent. The abscissa is radial direction outward to the right. The CMB is at $x=0.0$. The ordinate represents azimuthal direction. Flows along solid contours are cyclonic rolls (i.e. counterclockwise rotation). Flows along dashed contours are anticyclonic rolls (i.e. clockwise rotation). Temperature distribution is given as $\theta=\Delta T \cos 2 \pi y$ at $x=0$. The unit of the amplitude is $\alpha g \Delta T / \beta$. (a). For $\mathcal{E}=0.1, E=10^{-4}$. The contour interval is 0.15 . (b). For $\mathcal{E}=10, E=10^{-4}$. The contour interval is 0.02 . 
This situation is illustrated in Fig. 3(b).

The magnetic field can be calculated from Eq. (70) with the boundary conditions (71), when its dependence on $z$ is weak. The solution within the outer core is

$$
\begin{aligned}
g_{0}= & \frac{\alpha g \Delta T}{2 \lambda \beta} \frac{i l G}{1-i \mathcal{E}} e^{i l y}\left[\left(\frac{x}{l}-\frac{1}{2 l^{2}}\right) e^{l x}-\frac{1}{\left(k_{1}-i k_{2}\right)^{2}}\left(e^{\left(k_{1}-i k_{2}\right) x}-\frac{k_{1}-i k_{2}}{2 l} e^{l x}\right)\right. \\
& \left.-\frac{1}{\left(k_{2}+i k_{1}\right)^{2}}\left(e^{\left(k_{2}+i k_{1}\right) x}-\frac{k_{2}+i k_{1}}{2 l} e^{l x}\right)\right],
\end{aligned}
$$

and that within the mantle is

$$
g_{e x t, 0}=-\frac{\alpha g \Delta T}{4 \lambda \beta} \frac{i G}{1-i \mathcal{E}}\left[\frac{1}{l}-\frac{1}{k_{1}-i k_{2}}-\frac{1}{k_{2}+i k_{1}}\right],
$$

where we have used the relation $k_{1}, k_{2} \gg l$.

We solve $\phi$ in order to obtain the helicity. We have shown that $\phi_{0}=0$. The equation for $\phi_{1}$ is

$$
2 \Omega \frac{\partial}{\partial z} \triangle_{2} \phi_{1}=\left[-\nu \triangle_{2}^{2}+\frac{V_{A}^{2}}{\lambda} G^{2} \frac{\partial^{2}}{\partial y^{2}}\right] \psi_{0}-\alpha g \frac{\partial \theta}{\partial y} .
$$

From Eqs. (109) and (127), we have

$$
2 \Omega \frac{\partial}{\partial z} \triangle_{2} \phi_{1}=\left[\beta \frac{\partial}{\partial y}+\frac{V_{A}^{2}}{\lambda}\left(G^{2}-\left\langle G^{2}\right\rangle\right) \frac{\partial^{2}}{\partial y^{2}}\right] \psi_{0} .
$$

Integration in the $z$ direction gives

$$
\begin{aligned}
\triangle_{2} \phi_{1} & =\left(\frac{\beta}{2 \Omega} z \frac{\partial}{\partial y}+\frac{V_{A}^{2}\left\langle G^{2}\right\rangle L}{2 \Omega \lambda} f_{G G}(z) \frac{\partial^{2}}{\partial y^{2}}\right) \psi_{0} \\
& =-\frac{\alpha g \Delta T}{2 \Omega} \frac{i z-\mathcal{E} f_{G G}(z)}{1-i \mathcal{E}} l e^{i l y}\left(e^{l x}-\frac{1}{2} e^{\left(k_{1}-i k_{2}\right) x}-\frac{1}{2} e^{\left(k_{2}+i k_{1}\right) x}\right) .
\end{aligned}
$$

Helicity is obtained by substituting (113) and (130) into Eq. (96). Utilizing the approximation (103) again, we obtain

$$
\begin{aligned}
W_{1}(z)= & \frac{(\alpha g \Delta T)^{2}}{2 \beta \Omega} \frac{\mathcal{E} f_{G G}(z)}{1+\mathcal{E}^{2}} l^{3} L e^{2 l x} \\
& -\frac{(\alpha g \Delta T)^{2}}{8 \beta \Omega} \frac{k_{1} k_{2} l z}{1+\mathcal{E}^{2}}\left(e^{2 k_{1} x}-e^{2 k_{2} x}\right) \\
& +\frac{(\alpha g \Delta T)^{2}}{8 \beta \Omega} \frac{\mathcal{E} f_{G G}(z)}{1+\mathcal{E}^{2}} l L\left(k_{2} e^{2 k_{1} x}+k_{1}^{2} e^{2 k_{2} x}\right) .
\end{aligned}
$$

The results may be written symbolically as follows, as long as an order-of-magnitude estimate is concerned. The helicity in the interior region is

$$
W \sim \frac{(\alpha g \Delta T)^{2}}{2 \Omega \beta} l^{3} L \frac{\mathcal{E}}{1+\mathcal{E}^{2}},
$$

which is the same as the results of Subsection 2.1 (105). The helicity in the boundary layer is

$$
W \sim \begin{cases}\frac{(\alpha g \Delta T)^{2}}{8 \beta \Omega} l^{3} L \frac{E^{-1 / 2}}{1+\mathcal{E}^{2}} & \text { if } \quad \mathcal{E} \ll 1 \\ \frac{(\alpha g \Delta T)^{2}}{8 \beta \Omega} l^{3} L \frac{\mathcal{E}^{3 / 2} E^{-1 / 2}}{1+\mathcal{E}^{2}} & \text { if } \quad \mathcal{E} \gg 1\end{cases}
$$




\subsection{Summary of the linear response theory}

We summarize the results of our linear theory.

We assume that the Ekman number is small and that the poloidal field is much less than the toroidal field. These assumptions lead to the formation of a thin boundary layer. The flow outside the boundary layer (the interior region) is controlled by the magnitude of the Elsasser number.

When the Elsasser number is smaller than the order of unity, the flow is determined by the balance between the topographic $\beta$ effect and the thermal forcing; upwellings are located to the east of hot regions, and downwellings are located to the west of hot regions. The flow velocity is approximately given by

$$
\begin{aligned}
u & \sim \frac{\alpha g l \Delta T}{\beta} \\
& \sim 10^{-3} \mathrm{~m} / \mathrm{s}\left(\frac{\Delta T}{1 \mathrm{mK}}\right) .
\end{aligned}
$$

Here, we assumed that $l^{-1} \simeq L$ and $\beta \simeq \Omega / L$.

When the Elsasser number is larger than the order of unity, the flow is determined by the balance between the Lorentz force and the thermal forcing; upwellings are located beneath hot regions, and downwellings are located beneath cold regions. The flow velocity is approximately given by

$$
\begin{aligned}
u & \sim \frac{\alpha g l \Delta T}{\beta} \mathcal{E}^{-1} \\
& \sim 10^{-3} \mathrm{~m} / \mathrm{s}\left(\frac{\Delta T}{1 \mathrm{mK}}\right) \mathcal{E}^{-1}
\end{aligned}
$$

Limitation of the linear analysis is given by the Péclet number defined in terms of $u$ being less than the order of unity. If we adopt the estimate (135), the Péclet number is given by

$$
\begin{aligned}
P e & =u L / \kappa \\
& \sim\left(\frac{\Delta T}{1 \mathrm{mK}}\right)\left(\frac{10^{3} \mathrm{~m}^{2} / \mathrm{s}}{\kappa}\right) .
\end{aligned}
$$

If we tentatively take $\kappa$, the eddy diffusivity, to be $10^{2} \mathrm{~m}^{2} / \mathrm{s}, \Delta T$ must be less than about 0.1 $\mathrm{mK}$ in order that the Péclet number is less than the order of unity, and that the linear analysis is valid.

The helicity for the interior region is given approximately by

$$
W \sim \frac{(\alpha g \Delta T)^{2}}{2 \Omega \beta} l^{3} L \frac{\mathcal{E}}{1+\mathcal{E}^{2}} .
$$

The helicity within the boundary layer may be given approximately by

$$
W \sim \begin{cases}\frac{(\alpha g \Delta T)^{2}}{2 \Omega \beta} l^{3} L \epsilon^{-2} & \text { if } \quad \mathcal{E} \ll 1 \\ \frac{(\alpha g \Delta T)^{2}}{2 \Omega \beta} l^{3} L \epsilon^{-2} \mathcal{E}^{-1} & \text { if } \quad \mathcal{E} \gg 1,\end{cases}
$$

where

$$
\epsilon \equiv \text { (boundary layer thickness) } \times l \text {. }
$$




\section{Relation to the Observed Geomagnetic Field}

We apply the theoretical results to the Earth's core, although there are several limitations in the theoretical analysis as mentioned in Sections 1 and 2.

Bloxham and GubBins (1987) pointed out the importance of thermal coupling between the mantle and the outer core on the basis of the observation of the geomagnetic field. Here we reconsider the interpretation of observed features on the basis of the theory we have developed.

The most prominent static feature of the geomagnetic field is, according to GUBBINS and BloxнAm (1987), four lobes placed symmetrically about the equator. They interpreted them to be convection rolls parallel to the rotation axis and tangential to the inner core boundary. For the convection rolls with cyclonic rotation (i.e. positive relative vorticity), flow goes toward their centers in the Ekman layer, gathering magnetic lines of force to increase magnetic flux (e.g. Busse, 1970, 1975). For the convection rolls with anticyclonic rotation (i.e. negative relative vorticity), flow goes away from their centers in the Ekman layer, dispersing magnetic lines of force to decrease magnetic flux.

On the basis of our theory, we propose that these rolls are produced by temperature heterogeneity on the CMB (Fig. 4). According to recent seismological studies (e.g. DzIEWONSKI, 1984; TANimото, 1990; SU and DzIEwonski, 1991), a degree 2 sectorial pattern is predominant in the seismic velocity structure of the lower mantle. The regions with high velocity can be considered to be cold, and the regions with low velocity hot. We have shown that upwellings occur to the east of hot regions and downwellings occur to the west when the magnetic field is weak (i.e. $\mathcal{E} \ll 1$ ) and that upwellings occur beneath hot regions and downwellings occur beneath cold regions when the magnetic field is strong (i.e. $\mathcal{E} \gg 1$ ). As illustrated in Fig. 4 , the four flux lobes are located almost within the cold regions, where cyclonic rolls are expected to be generated when the magnetic field is weak, but a little shifted to the west. Hence, we conclude that the Elsasser number $\mathcal{E}$ is a little less than the order of unity. If the electric conductivity is $7 \times 10^{5} \mathrm{~S} / \mathrm{m}, \mathcal{E} \lesssim 1$ means that the toroidal field is a little less than about 10 gauss.

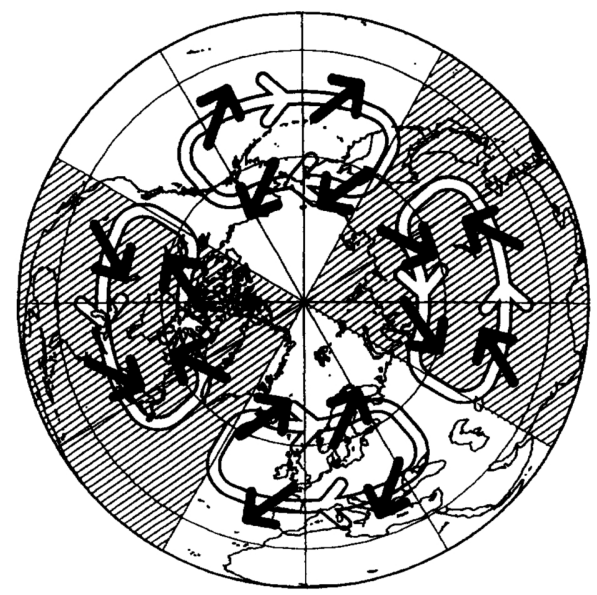

Fig. 4. Lower mantle heterogeneity and the flow induced by the temperature heterogeneity at the CMB. The hatched regions are high seismic-velocity regions of the lower mantle (SU and DzIEwONSKI, 1991), which can be supposed to be cold regions. The circulating arrows represent a cross section of convection rolls. The black arrows represent the flow in the Ekman layer, which is considered to be responsible for the concentration and dispersion of the magnetic flux. 


\section{Dynamo Action of a Thermally Driven Flow}

We investigate whether the flow derived in Section 2 can sustain dynamo action or not.

Busse (1975) showed that a magnetic field is generated when

$$
R_{\alpha} \equiv \frac{\alpha_{\text {dynamo }} \Lambda}{\lambda} \gtrsim 1,
$$

where $\alpha_{\text {dynamo }}$ is a typical magnitude of the $\alpha$ effect, $\Lambda$ the length scale, and $\lambda$ the magnetic diffusivity. The $\alpha$ effect is approximately given by

$$
\alpha_{\text {dynamo }} \simeq \frac{W \Lambda^{2}}{\lambda}
$$

where $W$ is the helicity.

The helicity calculated in Section 2 is summarized in Subsection 2.3. Assuming that $\Lambda \simeq$ $L \simeq l^{-1}$, we obtain the $R_{\alpha}$ for the interior region.

$$
R_{\alpha, \text { intr }} \sim R_{\alpha, 0} \frac{\mathcal{E}}{1+\mathcal{E}^{2}},
$$

where, $R_{\alpha, 0}$ is given by

$$
\begin{aligned}
R_{\alpha, 0} & \equiv\left(\frac{\alpha g \Delta T}{\lambda}\right)^{2} \frac{L}{2 \Omega \beta} \\
& \simeq\left(\frac{\Delta T}{1 \mu \mathrm{K}}\right)^{2} .
\end{aligned}
$$

For the boundary layer, $\Lambda$ should be taken as the boundary layer thickness,

$$
\Lambda \simeq \frac{\epsilon}{l} \simeq \epsilon L
$$

Consequently, the $R_{\alpha}$ for the boundary layer is given by

$$
R_{\alpha, \text { bdry }} \sim \begin{cases}R_{\alpha, 0} \epsilon & \text { if } \quad \mathcal{E} \ll 1 \\ R_{\alpha, 0} \epsilon \mathcal{E}^{-1} & \text { if } \quad \mathcal{E} \gg 1\end{cases}
$$

Comparing (145) with (149), we find that dynamo action is stronger in the interior region than in the boundary layer unless $\mathcal{E} \ll \epsilon$. Since we have shown in Section 3 that $\mathcal{E}$ is a little less than unity in the outer core, the dynamo action in the boundary layer would not be important. Thus we examine the dynamo action of the interior region.

The $R_{\alpha, \text { intr }}$ is illustrated in Fig. 5 as a function of the Elsasser number. On the other hand, studies of $\alpha^{2}$ dynamo have revealed that dynamo action occurs if $R_{\alpha}$ exceeds about unity. Since $\mathcal{E}$ is a little less than unity, the condition for dynamo generation for the flow is approximately given by

$$
R_{\alpha, 0} \gtrsim 1 \text {. }
$$

A temperature heterogeneity of about $1 \mu \mathrm{K}$ would suffice for dynamo generation.

We have shown that dynamo action is easily maintained by the flow driven by CMB temperature heterogeneities under a toroidal field of $\mathcal{E} \sim 1$. Hence, it is very likely that some portion of the dynamo action in the Earth's core is maintained by lateral temperature variations on the CMB. It is possible within the present theoretical framework that the whole dynamo action is maintained by CMB temperature heterogeneities, but some difficulty might arise in relation to thermal efficiency (e.g. Gubbins and Masters, 1979). 


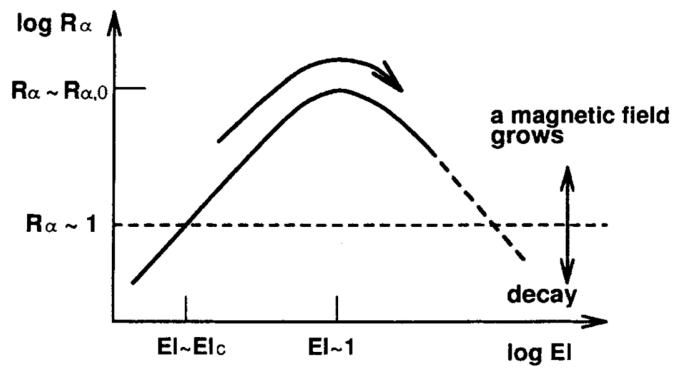

Fig. 5. Schematic diagram of dynamo action. The abscissa represents the Elsasser number, a measure of the strength of the magnetic field. The ordinate is the magnetic Reynolds number, a measure of the magnitude of the helicity. The curve represents the magnetic Reynolds number in the interior region as a function of the Elsasser number. The helicity increases as the magnetic field increases from zero, and reaches a maximum when the Elsasser number is of the order of unity. On the other hand, when the magnetic Reynolds number exceeds the order of unity, the magnetic field grows as a result of dynamo action. Therefore, if the Elsasser number is grater than the critical value $E l_{c}$, the magnetic field grows.

\section{Conclusions}

We have investigated the linear response of the outer core to the sectorial temperature heterogeneity on the CMB. We have shown that the positions of upwelling and downwelling are strongly affected by the strength of the magnetic field; under a weak magnetic field, upwellings and downwellings occur between hot and cold regions as ZHANG and GuBBINS (1992) calculated, while under a strong magnetic field, upwellings occur beneath hot regions and downwellings occur beneath cold regions as Bloxham and GubBins (1987) suggested.

We have inferred that the Elsasser number is less than unity in the outer core by comparing the theory with the observed magnetic field.

It is shown that dynamo action would be maintained by the flow driven by a temperature heterogeneity of the order of more than $10^{-6} \mathrm{~K}$ on the CMB.

We thank M. Kumazawa for his continuous encouragement over the course of the study. We are grateful to S. I. Braginsky, J. Bloxham and I. Sumita for helpful comments on the manuscript.

\section{REFERENCES}

Bloxнam, J. and D. Gubbins, Thermal core-mantle interactions, Nature, 325, 511-513, 1987.

Busse, F. H., Thermal instabilities in rapidly rotating systems, J. Fluid Mech., 44, 441-460, 1970.

Busse, F. H., A model of the geodynamo, Geophys. J. R. astr. Soc., 42, 437-459, 1975.

Busse, F. H., Generation of planetary magnetism by convection, Phys. Earth Planet. Inter., 12, 350-358, 1976.

DzIEwonski, A. M., Mapping the lower mantle: determination of lateral heterogeneity in $\mathrm{P}$ velocity up to degree and order 6, J. Geophys. Res., 89, 5929-5952, 1984.

Gubbins, D. and J. Bloxham, Morphology of the geomagnetic field and implications for the geodynamo, Nature, 325, 509-511, 1987.

Gubbins, D. and T. G. Masters, Driving mechanisms for the Earth's dynamo, Adv. Geophys., 21, 1-50, 1979.

HidE, R., Free hydromagnetic oscillations of the Earth's core and the theory of the geomagnetic secular variation, Phil. Trans. R. Soc. Lond., A259, 615-647, 1966.

Mansinha, L., D. E. Smylie, and B. Sctherland, Earthquakes and the spectrum of the Brussels Superconducting Gravimeter data for 1982-1986, Phys. Earth Planet. Inter., 61, 141-148, 1990.

MelchioR, P. and B. DuCARME, Detection of inertial gravity oscillations in the Earth's core with a superconducting gravimeter at Brussels, Phys. Earth Planet. Inter., 42, 129-134, 1986.

Pedlosky, J., Geophysical Fluid Dynamics, 2nd ed., 710 pp., Springer-Verlag, New York, 1987.

$\mathrm{Su}$, W.-J. and A. M. DzIEwONSKI, Predominance of long-wavelength heterogeneity in the mantle, Nature, 352, $121-126,1991$ 
TAnimoto, T., Long-wavelength S-wave velocity structure throughout the mantle, Geophys. J. Int., 100, 327-336, 1990.

YANO, J.-I., Asymptotic theory of thermal convection in rapidly rotating system, J. Fluid Mech., 243, 103-131, 1992.

ZhANG, K. and D. GLBBINS, On convection in the earth's core driven by lateral temperature variations in the lower mantle, Geophys. J. Int., 108, 247-255, 1992.

Z̈̈rn, W., B. Richter, P. Rydelek, and J. Neuberg, Comment--Detection of inertial gravity oscillations in the Earth's core with a superconducting gravimeter at Brussels, Phys. Earth Planet. Inter., 49, 176-178, 1987. 